\title{
Larva de Rhynchophorus palmarum L. (Coleoptera: Curculionidae): Efecto de la dieta en la síntesis de ácidos grasos esenciales
}

\section{Larva of Rhynchophorus palmarum L. (Coleoptera: Curculionidae): Effect of diet on the synthesis of essential fatty acids}

\author{
Julio Cesar Maceda Santivañez $\mathbb{1}^{1}$, Larry Oscar Chañi Paucar $\mathbb{D}^{2}$
}

${ }^{1}$ Ingeniero Agroindustrial, Escuela profesional de Ingeniería Agroindustrial, Universidad Nacional Amazónica de Madre de Dios, Av. Jorge Chávez 1160, Puerto Maldonado, Madre de Dios, Perú. Postgraduando en Biotecnologia y Recursos Naturales, Universidade do Estado do Amazonas, Escola Superior de Ciências da Saúde Av. Carvalho Leal 1777, Manaus, Amazonas, Brasil. cesantiv@gmail.com. ${ }^{2} \mathrm{PhD}$ student in food engineering, Graduate Program on Food Engineering, School of Food Engineering, University of Campinas (UNICAMP), R. Monteiro Lobato 80, 13083-862 Campinas, SP, Brazil. Larry.76728@ gmail.com.

\section{A R T I G O}

Recebido: 08/07/2020

Aprovado: 22/02/2021

Palavras-chave:

Alimentos exóticos

Oviposición

Larvas comestibles

Lípidos
Larvicultura

\section{R E S U M E N}

Los insectos son fuente importante de proteínas, vitaminas, aminoácidos, minerales y lípidos. En ese contexto el presente estudio, evaluó el perfil de ácidos grasos esenciales del aceite de larva de Rhynchophorus palmarum (Coleoptera: Curculionidae) sometida a tres dietas de tejidos vegetales: Mauritia flexuosa (Arecaceae), Oenocarpus bataua (Arecaceae) y Jacaratia digitata (Caricaceae). Fueron capturados cinco individuos adultos, 2 machos y 3 hembras para su reproducción en cautiverio. Las larvas eclosionadas se sometieron a las tres dietas por 45 días. Después de este periodo se efectuó la extracción y se determinó el perfil de ácidos grasos del aceite. Siendo el ácido graso monoinsaturado oleico (18:1) y el ácido graso saturado palmítico (16:0), los que se encuentran en mayor abundancia para las tres dietas. Respecto a los ácidos grasos esenciales, fueron detectados los ácidos grasos linoleico (18:2) y alpha linolénico (18:3) en concentraciones de 1,96 \pm 0,028 y 1,14 $\pm 0,007 \%$, para la dieta de Mauritia flexuosa, $0,67 \pm 0,007$ y 0,43 $\pm 0,035 \%$, para la dieta de Oenocarpus bataua, y 0,76 $\pm 0,000$ y 0,29 $\pm 0,007 \%$, para la dieta de Jacaratia digitata, respectivamente. Los resultados muestran que las dietas influyen en el tipo de ácido graso metabolizado y su concentración. La larva es fuente de ácidos grasos monoinsaturados como el oleico (omega 9) y el palmitoleico (omega 7), y de los ácidos grasos esenciales linoleico y alpha linolénico precursores de los ácidos grasos de la familia omega 6 y omega 3.

\section{A B S T R A C T}

Key words:

Exotic foods

Oviposition

Larviculture

Edible larvae

Lipids
Insects are an important source of proteins, vitamins, amino acids, minerals, and lipids. In this context, the present study evaluated the essential fatty acid profile of the larval oil of Rhynchophorus palmarum (Coleoptera: Curculionidae) subjected to three plant tissue diets: Mauritia flexuosa (Arecaceae), Oenocarpus bataua (Arecaceae), and Jacaratia digitata (Caricaceae). Five adult individuals, 2 males, and 3 females were captured for their reproduction in captivity. The hatched larvae were subjected to the three diets for 45 days. After this period, extraction was carried out and the fatty acid profile of the oil was determined. Being oleic monounsaturated fatty acid (18:1) and palmitic saturated fatty acid (16:0), which are found in greater abundance for the three diets. Regarding essential fatty acids, linoleic (18:2) and alpha-linolenic (18:3) fatty acids were detected in concentrations of $1.96 \pm 0.028$ and $1.14 \pm 0.007 \%$, for the diet of Mauritia flexuosa, $0.67 \pm 0.007$ and $0.43 \pm 0.035 \%$, for the diet of Oenocarpus bataua, and $0.76 \pm 0.000$ and $0.29 \pm 0.007 \%$, for the diet of Jacaratia digitata, respectively. The results show that diets influence the type of fatty acid metabolized and its concentration. The larva is a source of monounsaturated fatty acids such as oleic (omega 9) and palmitoleic (omega 7) and of the essential fatty acids linoleic and alpha-linolenic precursors of the fatty acids of the omega 6 and omega 3 families.

\author{
Revista Verde \\ ISSN 1981-8203 \\ Pombal, Paraíba, Brasil
}

v. 16, n.2, abr.-jun, p.122-130, 2021

doi: $10.18378 /$ rvads.v16i2.8258 


\section{INTRODUCCIÓN}

El consumo de insectos se practica en muchos países del mundo, principalmente en Asia, África, América y pueblos aborígenes australianos. La evidencia arqueología sugiere que la entomofagia (consumo de insectos por los seres humanos) se ha practicado desde la aparición de los seres humanos. Los insectos están incluidos en la dieta regular de cerca de 2 billones de personas, con un aproximado de 2000 especies de insectos comestibles. El uso de insectos como alimento humano puede ser por ingesta de sus huevos, larvas, pupas, así como del animal adulto (COSTA-NETO, 2013; FAO, 2013).

El mayor grupo de insectos comestibles son los coleópteros $(31 \%)$, principalmente los escarabajos en su etapa larvaria, lepidópteros (17\%), como orugas, himenópteros (15\%), entre avispas, abejas y hormigas, Orthopteras (14\%), entre grillos, saltamontes y langostas, Hemipteras (11\%), como chinches, Isopteras (3\%), como termitas, y el resto entre Odonata y díptera (9\%) como libélulas y moscas respectivamente. (VAN HUIS, 2019).

Según Costa-Neto (2011), el hábito de comer insectos puede ser promovida a través de la educación, enfatizando en los beneficios nutricionales que los insectos comestibles pueden proporcionar.

La cría de insectos es uno de los caminos para la seguridad alimentaria, presentándose como una alternativa barata y sostenible, pudiendo generar oportunidades empresariales en las economías desarrolladas, en fase de transición y en desarrollo (FAO, 2013). Según Defoliart (1995), los insectos son criados de modo más eficiente que los bovinos, cerdos, aves y peces.

En Japón la especie Oxya yezoensis es comercializada enlatada, cocida en salsa de soja y azúcar, en Tailandia se puede encontrar insectos enlatados como: grillos cocidos, pupa de gusano de seda, y larvas de bambú. Productos a base de insectos se pueden encontrar en restaurantes y supermercados en Europa, Estados Unidos y Brasil (FAO, 2013; FAPESP, 2020).

La empresa francesa Jimini's produce barra de cereales, pasta y granola a base de insectos, además de bocaditos de insectos deshidratados y sazonados. En Estados Unidos, la empresa Chirps importa materia prima de Tailandia para la elaboración de harina, snacks y galletas. En Alemania, BugFoundation vende hamburguesas que llevan un $45 \%$ de una mixtura proteica a base de soya y larva de escarabajo (Alphitobius diaperinus) (FAPESP, 2020).

Los insectos proporcionan nutrientes de alta calidad en comparación con la carne, pescado, y presentan un riesgo reducido de transmisión de enfermedades zoonóticas (enfermedades transmitidas de los animales a los humanos (FAO, 2013).

Los insectos son ricos en aminoácidos, lípidos, vitaminas, minerales y muchos cumplen con los requisitos de aminoácidos para los humanos, son altos en ácidos grasos monoinsaturados, ácidos grasos poliinsaturados, dentro los minerales tenemos, cobre, hierro, magnesio, manganeso, fósforo, selenio y zinc, vitaminas como riboflavina, ácido pantoténico, biotina y en algunos casos ácido fólico (RUMPOLD; SCHLÜTER, 2013; VAN HUIS, 2013).
Existe un interés creciente por los insectos, no solo para consumo humano, sino como fuente alternativa de proteínas para la producción de alimento para animales, pues los insectos pueden complementar las fuentes de alimentos tradicionales como la soja, el maíz y la harina de pescado (VAN HUIS, 2013).

El contenido de proteína sobre una base de materia seca de los insectos varía entre 7 y 91\% (FINKE; OONINCX, 2013).

Los lípidos son considerados como buena fuente de energía, vitaminas liposolubles (A, D, E y K) y de ácidos grasos esenciales. Los ácidos grasos esenciales no pueden ser sintetizados por el organismo humano y tienen que ser suministrados a través de la dieta, su ausencia en el organismo podría producir diversos trastornos como: dermatitis, mala cicatrización de las heridas, coagulación sanguínea dificultosa, entre otras (CALDER, 2011; ESHAK et al., 2018).

Entre los ácidos grasos sólo los de la familia omega 6 (n6) y omega $3 \quad(n-3)$ son esenciales. Los principales representantes de la familia omega 6 son: ácido linoleico o LA (18:2n-6), ácido araquidónico o ARA (20:4n-6), y de la familia omega 3 son: ácido alpha linolénico o ALA (18:3n-3), ácido eicosapentaenóico o EPA (20:5n-3) y el ácido docosahexaenóico o DHA (22:6n-3) (SIMOPOULOS, 2016; WATANABE; TATSUNO, 2020).

Los ácidos grasos de cadena larga de las familias $n-6$ y n-3 son fundamentales en la función, estructura, fluidez y actividad proteica de la membrana celular (ANTONNY et al., 2015; BIGAY; ANTONNY, 2012). Son necesarios para mantener bajo condiciones normales, las funciones cerebrales y la transmisión de impulsos nerviosos. Estos también participan en la transferencia de oxígeno atmosférico para el plasma sanguíneo, en la síntesis de la hemoglobina y división celular (YEHUDA et al., 2002).

El ácido araquidónico (ARA) y el docosahexaenoico (DHA) son fundamentales en la formación de la estructura y en la funcionalidad del sistema nervioso y visual de los humanos, constituyendo más del $30 \%$ de la estructura lipídica del cerebro y de la retina (VALENZUELA; NIETO, 2003). Así también el ácido graso eicosapentaenoico (EPA), junto con el ácido docosahexaenoico (DHA) son generadores de lipídicos bioactivos mediadores importantes en la resolución de la inflamación (WEYLANDT et al., 2012).

Los ácidos grasos de las familias n-6 y n-3 son obtenidos por medio de la dieta o producidos por el organismo a partir de los ácidos linoleico y alfa-linolénico mediante la acción de las enzimas enlongasa y desaturasa (FABIAN et al., 2015).

En la región Madre de Dios, así como en otras regiones amazónicas del Perú, existe una gran diversidad de alimentos exóticos con características muy peculiares, que constituyen la dieta del poblador amazónico, entre las cuales se destaca la larva de Rhynchophorus palmarum L. (Coleóptera: Curculionidae). La larva es fuente de energía y proteína, y su consumo también fue reportado en poblaciones indígenas amazónicas en Ecuador, Colombia y Venezuela (CARTAY, 2018; DUE et al., 2009; SANCHO et al., 2015; VARGAS et al., 2013).

En la Amazonía peruana, las larvas de $R$. palmarum son extraídas principalmente del estípite de la palmera Aguaje 
(Mauritia flexuosa). Esta especie posee un alto valor económico, pues de ella se obtiene su fruto y derivados de la misma (KAHN; MEJÍA, 2006), altamente demandados, tanto en las regiones amazónicas, así como en diversas regiones del Perú.

Con la finalidad de masificar la producción de esta larva, Delgado et al. (2008), propuso el empleo de palmeras "macho" de $M$. Flexuosa para su producción en áreas con sobre población de palmeras "macho". Esto debido a la tala de palmeras "hembras" para la obtención de su fruto y así liberar áreas para plántulas en crecimiento, no en tanto, esto también podría generar problemas en las poblaciones naturales.

El $R$. palmarum es un insecto polífago que afecta a una gran variedad de especies, donde predomina la familia Arecaceae, así como: Caricaceae; Gramineae; Musaceae; Bromeliaceae; Anacardiaceae; Annonaceae; Lauraceae; Rutaceae y Moraceae (QUEIROZ et al., 2013). Estas son fuentes de alimento para los adultos, que pueden ser tomadas en consideración al momento de implementar su cría, así como también los residuos orgánicos de especies pertenecientes a estas familias, pues algunas especies de insectos pueden cultivarse en residuos orgánicos, y así contribuir a la disminución de la contaminación ambiental (VELDKAMP et al., 2012).

En tal sentido, el presente estudio tuvo como objetivo evaluar el perfil de ácidos grasos esenciales del aceite de las larvas de Rhynchophorus palmarum sometida a tres dietas de tejidos vegetales: Mauritia flexuosa (Arecaceae), Oenocarpus bataua (Arecaceae) y Jacaratia digitata (Caricaceae).

\section{MATERIAL Y MÉTODOS}

La investigación se realizó en las instalaciones del Laboratorio de Procesos Agroindustriales, de la Universidad Nacional Amazónica de Madre de Dios, ubicada en la ciudad de Puerto Maldonado, Madre de Dios-Perú.

Los especímenes adultos de $R$. palmarum fueron capturados en la comunidad de Bajo Madre de Dios (E: 0488185, W: 8606283), provincia y distrito de Tambopata, región Madre de Dios-Perú (Figura 1).

La captura fue realizada utilizando como atrayente el tejido fresco del estípite de la palmera Attalea phalerata (Arecaceae) conocido con el nombre común de Shapaja. El tejido fue colocado en envases plásticos por un tiempo de $48 \mathrm{~h}$, donde los especímenes adultos fueron atraídos, capturados manualmente y colocadas en frascos de vidrio con tapas perforadas, capturándose 5 especímenes (3 hembras y 2 machos), consiguiendo diferenciarlos pues, los machos presentan un penacho de pelos en el rostrum (pico), las hembras tienen el rostrum curvo y liso características muy visibles (MEXZÓN et al., 1994).

Figura 1. Ubicación de la comunidad de Bajo Madre de Dios provincia y distrito de Tambopata, región Madre de Dios.

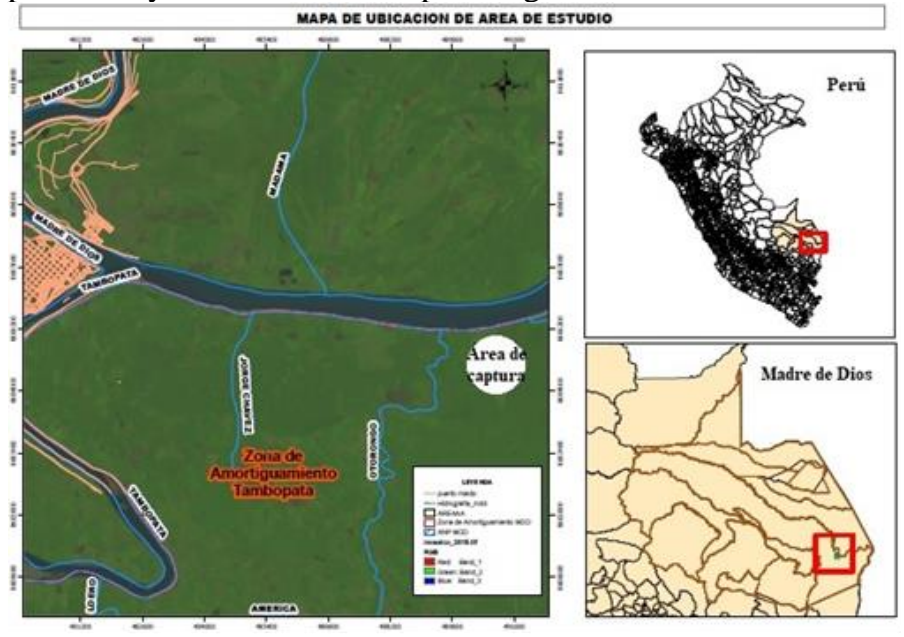

Fuente: Autores (2020).

La oviposición se llevó a cabo dentro de una caja de madera con dimensiones de 0,20 $\mathrm{m}$ de ancho, 0,20 $\mathrm{m}$ de largo y $0,25 \mathrm{~m}$ de alto. Esta caja fue construida con un lado de cristal transparente para permitir el monitoriamente visual y una de malla fina para permitir el flujo de aire (Figura 2). Los especímenes adultos de $R$. palmarum fueron colocados en la caja de oviposición con $400 \mathrm{~g}$ de tejido vegetal por un periodo de 24 h por cada tipo de tejido vegetal: M. flexuosa, O. bataua y J. digitata.

Los tejidos vegetales ovipositados se colocaron en cajas de cristal con dimensiones de $0,18 \mathrm{~m}$ de ancho, 0,20 $\mathrm{m}$ de largo $\mathrm{y}$ $0,10 \mathrm{~m}$ de altura y fueron cubiertas con material plástico para evitar la resequedad de las mismas por un periodo de 14 días.

Todo el experimento fue llevado a cabo en condiciones de temperatura ambiente, entre $30-33^{\circ} \mathrm{C}$ y humedad relativa entre $71-74 \%$.

Figura 2. R. palmarum ovipositando en tejido vegetal de $M$. flexuosa (A), O. bataua (B) y J. digitata (C).
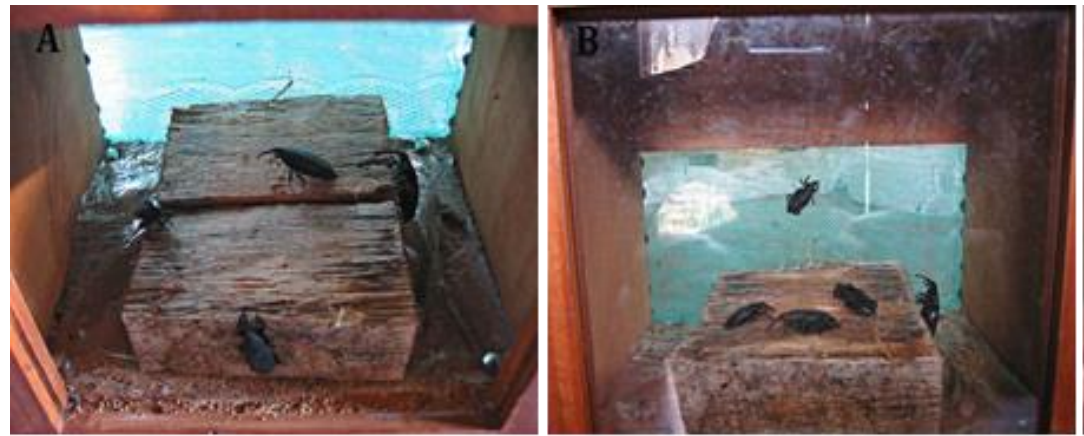

Fuente: Autores (2020)

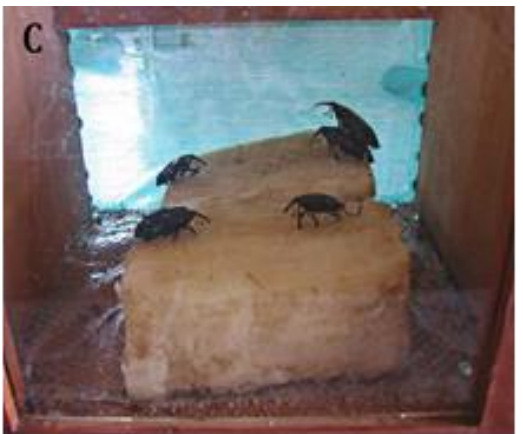


Durante este periodo de 14 días, se llevó a cabo la eclosión de los huevos y los primeros días de crecimiento larvario, para después ser extraídas (Figura 3). Fueron seleccionadas 21 larvas por cada tipo de dieta (tejido vegetal de M. flexuosa, O. bataua y $J$. digitata), buscando uniformidad de tamaño y buen estado físico (sin lesiones aparentes), sumando 63 larvas.

Cada larva seleccionada fue inmediatamente colocada en $200 \mathrm{~g}$ de su respectiva dieta (tejido vegetal de M. flexuosa, $O$. bataua y J. digitata), y distribuidas individualmente en cajas de cristal de dimensiones $0,11 \mathrm{~m}$ de ancho, $0,18 \mathrm{~m}$ de largo y 0,10 $\mathrm{m}$ de altura. Cada dieta fue renovada entre 2 y 3 días, dependiendo de la velocidad de alimentación de cada larva. La etapa de cría duro un periodo de 45 días, contados a partir de finalizado la oviposición.

Figura 3. Huevos (A) y larvas de 14 días de cría (B) de $R$. palmarum.
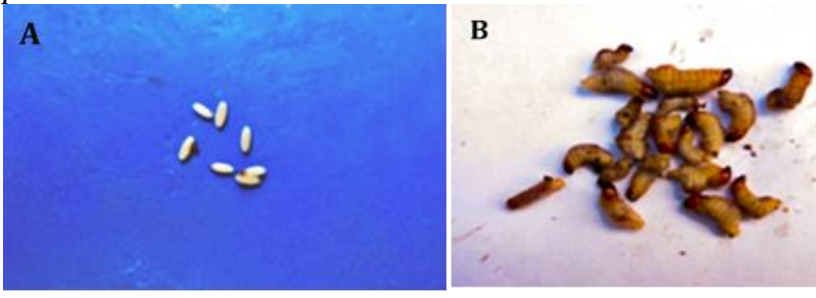

Fuente: Autores (2020).

Finalizada la etapa de cría se seleccionaron 15 larvas, formando tres grupos de 5 larvas por cada tipo de dieta (repeticiones), sumando 45 larvas y 9 grupos. Cada grupo fue sometida a las siguientes operaciones de acondicionamiento: descabezado, eviscerado, reducción de tamaño del cuerpo graso a $0,5 \mathrm{~cm}^{2}$, secado en estufa (Tomos ODGH-9053) a $50{ }^{\circ} \mathrm{C}$ por 3 $\mathrm{h}$ y enfriado en campanas desecadoras por $30 \mathrm{~min}$. Para después ser sometido a extracción empleando el método de Soxhlet (TZOMPA-SOSA et al., 2014), utilizando como solvente hexano $\left(\mathrm{C}_{6} \mathrm{H}_{14}\right)$ al $98,5 \%$, la extracción fue llevada a cabo por 90 min. El contenido graso obtenido se llevó a estufa a $68{ }^{\circ} \mathrm{C}$ por $5 \mathrm{~h}$, con la finalidad de eliminar restos del solvente, seguidamente fue sometido a enfriamiento en campana desecadora, envasada y almacenada en frascos de vidrio ámbar de $2 \mathrm{~mL}$ a $-20{ }^{\circ} \mathrm{C}$, para análisis posterior.

Las dietas fueron analizadas siguiendo las orientaciones de la AOAC, para humedad (AOAC 930.04 2005), Cenizas (AOAC 930.05 2005), Proteína (978.04 (A) 2005), y las normas técnicas peruanas para Fibra (NTP 250.003 1980), Azucares reductores totales (NTP 290.173 1999), Grasa cruda (NTP 250.041 1976) y Carbohidratos por diferencia.

El perfil de ácidos grasos del aceite fue determinado empleando un cromatógrafo de gases (Perkin Elmer Autosystem XL) acoplado a un detector de ionización de flama (FID). Previamente al análisis las muestras de aceite fueron esterificadas con una solución metanólica de hidróxido de potasio (RUIZ et al., 2013). Las condiciones de análisis fueron: Temperatura del horno de $160-230{ }^{\circ} \mathrm{C}\left(1{ }^{\circ} \mathrm{C} / \mathrm{min}\right)$, temperatura del inyector de $250{ }^{\circ} \mathrm{C}$, temperatura del detector de $270{ }^{\circ} \mathrm{C}$, presión de hidrógeno de 5 psi, Split de 100:1 y un volumen de inyección de $2 \mu$ l. Tipo de columna Supelcowax-10 de sílice fundida (Supelco) de $30 \mathrm{~m}$ de longitud, $0,25 \mathrm{~mm}$ de diámetro interno y $0,25 \mu \mathrm{m}$ de espesor de película, y un tiempo de análisis de $65 \mathrm{~min}$.

Para el análisis estadístico se utilizó el programa estadístico Sisvar 5.6. Se aplicó un análisis de varianza (ANOVA) con un nivel de significancia del 5\%, los pares de medias fueron comparados mediante la prueba de Tukey $(\mathrm{p}<0,05)$.

\section{RESULTADOS Y DISCUCIONES}

La Tabla 1 muestra la composición proximal y el valor calórico de los tejidos internos de los estípites de M. flexuosa, O. bataua y $J$. digitata, utilizados como alimentos para las larvas de $R$. palmarum, se puede observar que los componentes mayoritarios son: agua y carbohidratos, siendo el tejido de $J$. digitata el que presento mayor contenido de humedad, en contraste fue el que presento el menor contenido de carbohidratos, hecho que influiría en la disponibilidad de energía calórica, pudiendo esto comprometer el crecimiento y desarrollo de las larvas (GIBLIN-DAVIS et al., 1989).

Tabla 1. Composición química proximal ( $100 \mathrm{~g})$ de los tejidos vegetales de $M$. flexuosa, O. bataua y J. digitata.

\begin{tabular}{lccc}
\hline \multirow{2}{*}{ Composición } & \multicolumn{3}{c}{ Tejido vegetal } \\
\cline { 2 - 4 } & $\begin{array}{c}M . \\
\text { flexuosa }\end{array}$ & $\begin{array}{c}\text { O. } \\
\text { bataua }\end{array}$ & $\begin{array}{c}J . \\
\text { digitata }\end{array}$ \\
\hline Humedad $(\mathrm{g})$ & 67,2 & 69,5 & 94,1 \\
Proteína cruda $(\mathrm{g})$ & 0,6 & 0,6 & 0,3 \\
Grasa cruda $(\mathrm{g})$ & 0,1 & 0,1 & 0,1 \\
Cenizas totales $(\mathrm{g})$ & 0,5 & 0,3 & 1,1 \\
Carbohidratos $(\mathrm{g})$ & 31,6 & 29,5 & 4,4 \\
Fibra cruda $(\mathrm{g})$ & 3,9 & 4,1 & 1,6 \\
Energía (Kcal) & 129,7 & 121,3 & 19,7 \\
Azucares reductores $(\mathrm{g})$ & 0,0 & 0,0 & 0,0 \\
\hline
\end{tabular}

El contenido graso observado en las larvas alimentadas con tejido del estípite de M. flexuosa, O. bataua y J. digitata (Tabla 2) fueron próximos a lo reportado por Gbogouri et al. (2013), que obtuvo $21,8 \%$. Vargas et al. (2013), en un estudio con larvas extraídas del medio natural del estípite de $M$. flexuosa y comercializadas en el mercado de la ciudad de Belén de Iquitos-Perú, obtuvo un contenido graso de 22,11 y 44,30\%, para la piel y el tracto digestivo respectivamente. En otro estudio con larvas de $R$. palmarum extraídas de estípites de palmeras nativas en Costa de Marfil, mostraron un contenido graso de 35 y $49 \%$ (DUE et al., 2009).

Las larvas sometidas a la dieta de M. flexuosa y O. bataua evidenciaron mayor contenido graso, y presentaron diferencia significativa $(\mathrm{p}<0,05)$, respecto a las larvas sometidas a la dieta $J$. digitata (Tabla 2). Asimismo, se puede observar que el contenido graso de las larvas alimentadas con los diferentes tejidos vegetales presenta una correlación positiva respecto al contenido de carbohidratos ( $\mathrm{r}_{\text {Pearson }}=0,980$ ), y de energía calórica $\left(r_{\text {Pearson }}=0,980\right)$ de las dietas (Tabla 1$)$.

La correlación positiva observada entre el contenido de carbohidratos, energía de las dietas y el contenido lipídico de las larvas de $R$. palmarum, puede explicarse debido a que el 
metabolismo de las larvas les permite almacenar la energía calórica en la forma de compuestos lipídicos, especialmente triglicéridos, sintetizados a partir de los nutrientes disponibles en las dietas suministradas, especialmente los carbohidratos (SANTANA et al., 2014). Según Angel-Dapa et al. (2010), en los insectos, los lípidos juegan un papel clave en la maximización de la tasa de huevos eclosionados (fecundidad), crecimiento larvario y supervivencia.

Tabla 2. Contenido graso de las larvas de $R$. palmarum

\begin{tabular}{lc}
\hline Larva por fuente alimentícia & Grasa $(\%)$ \\
\hline M. flexuosa & $24,40 \pm 0,413 \mathrm{a}$ \\
O. bataua & $23,04 \pm 0,825 \mathrm{a}$ \\
J. digitata & $19,42 \pm 0,421 \mathrm{~b}$ \\
\hline
\end{tabular}

Datos expresados en promedio \pm desviación estándar.

Medias seguidas de letras iguales no son significativamente diferentes por la prueba de Tukey al 5\% de probabilidad.

En los datos reportados en la tabla 3 se observa el peso y longitud de las larvas sometidas a las tres dietas a los 14 días, etapa en la cual se buscó homogeneidad de las larvas en los tratamientos, donde la variable Peso, no presenta diferencia estadística significativa $(\mathrm{p}<0,05)$, mientras que, la variable Longitud evidencia diferencia estadística significativa $(\mathrm{p}<0,05)$ entre las dietas J. digitata y O. bataua.
Para los datos obtenidos a los 45 días de cría (Tabla 3), se observa diferencia estadística significativa $(\mathrm{p}<0,05)$, entre las dietas $M$. flexuosa y $O$. bataua para la variable Peso. Respecto a la variable Longitud, entre las dietas $J$. digitata y O. bataua. Datos que fueron inferiores a lo encontrado en la literatura (DELGADO et al., 2008; MEXZÓN et al., 1994).

En el presente estudio, la etapa de cría se contabilizó concluida la oviposición en los tejidos vegetales, donde los huevos permanecieron dentro del tejido vegetal sin poder visualizar el momento de la eclosión de las mismas, y considerando que la fase de huevo en promedio es 3,5 días (MEXZÓN et al., 1994), resultando así un periodo de 41,5 días, siendo el desarrollo larvario entre 50 a 70 días (DELGADO et al., 2008), este hecho podría explicar los parámetros de peso y longitud observadas en las larvas concluido el periodo de cría (Tabla 3).

Es probable que, al aumentar el periodo de cría, se podría alcanzar mayor peso, longitud y contenido graso, como lo reportado en estudios anteriores. Estos parámetros en los insectos se ven influenciados por la etapa de desarrollo larvario, la calidad nutritiva de la dieta suministrada, y el medio ambiente (temperatura, humedad y fotoperíodo). Así también, factores ambientales podrían explicar la mortandad observada (Tabla 3), donde las larvas sometidas a la dieta de J. digitata, presentaron la tasa de mortalidad más baja (FINKE; OONINCX, 2013).

Tabla 3. Peso, longitud y mortalidad de las larvas de $R$. palmarum.

\begin{tabular}{llccc}
\hline \multirow{2}{*}{ Tiempo } & Parámetro & \multicolumn{1}{c}{ Dieta } \\
\cline { 2 - 5 } 14 días & Peso $(\mathrm{g})$ & $0,46 \pm 0,086 \mathrm{a}$ & $0,40 \pm 0,041 \mathrm{a}$ & $0,36 \pm 0,007 \mathrm{a}$ \\
& Longitud $(\mathrm{cm})$ & $0,62 \pm 0,047 \mathrm{ab}$ & $0,53 \pm 0,024 \mathrm{~b}$ & $0,72 \pm 0,024 \mathrm{a}$ \\
\hline \multirow{2}{*}{45 días } & Peso $(\mathrm{g})$ & $3,81 \pm 0,209 \mathrm{a}$ & $2,78 \pm 0,317 \mathrm{~b}$ & $3,42 \pm 0,218 \mathrm{ab}$ \\
& Longitud $(\mathrm{cm})$ & $3,10 \pm 0,170 \mathrm{ab}$ & $2,40 \pm 0,216 \mathrm{~b}$ & $3,30 \pm 0,386 \mathrm{a}$ \\
\hline
\end{tabular}

Datos de peso y longitud expresados en promedio \pm desviación estándar.

Medias seguidas de letras iguales entre filas no son significativamente diferentes por la prueba de Tukey al $5 \%$ de probabilidad.

La tabla 4 muestra la composición de ácidos grasos de la larva de $R$. palmarum. El contenido de ácidos grasos saturados de este estudio, difiere respecto a lo reportado por otros autores donde sólo reportan los ácidos grasos mirístico (14:0), palmítico (C16:0), y esteárico (18:0) en concentraciones próximas (DUE et al., 2009; GBOGOURI et al., 2013; VARGAS et al., 2013). Sancho et al. (2015), reporta sumado a los tres anteriormente mencionados, el ácido graso láurico (12:0), ácido graso encontrado en este estudio, sólo en larvas sometidas a la dieta de $J$. digitata, Así mismo, reporta el ácido graso palmítico (C16:0), en una concentración inferior (28\%).

En el presente estudio, los ácidos grasos caprílico (8:0), cáprico (10:0) y láurico (12:0), sólo fueron detectados en las larvas sometidas a la dieta de $J$. digitata, y el ácido graso heptadecaenoico (17:0) únicamente fue detectado en las larvas sometida a la dieta de $M$. flexuosa.

El ácido graso monoinsaturado oleico (C18:1), en estudios similares fue reportado en concentraciones de 46,71 y 59,20\% (DUE et al., 2009; SANCHO et al., 2015), superior a lo obtenido en este estudio, también concentraciones próximas fueron reportados por Vargas et al. (2013) y Gbogouri et al.
(2013), con 41,57 y 44,6\%, respectivamente. Estos datos permiten considerar el aceite de larva de $R$. palmarum como buena fuente de este. El ácido oleico es importante en nuestra dieta, pues ayuda a la reducción plasmática de las lipoproteínas de baja densidad (LDL) y colesterol total, presentando efectos benéficos para la salud humana reduciendo el riesgo de enfermedades cardiovasculares (JORIS; MENSINK, 2016; VAFEIADOU et al., 2015).

El ácido graso monoinsaturado palmitoleico (16:1), fue reportado en concentraciones de $1,01, \quad 1,20$ y $2,10 \%$ (GBOGOURI et al., 2013; SANCHO et al., 2015; VARGAS et al., 2013), próximos a lo obtenido en esta investigación. Este ácido graso viene teniendo una notable importancia en los últimos años, pues se le atribuyen una serie de beneficios, al ser un potente antinflamatorio, aumenta la expresión genética de PPAR- $\alpha$ (Receptor activado por proliferadores de peroxisoma alfa), es un inhibidor de NFkB (factor nuclear kappa B), reconocido por aumentar la inflamación celular (SOUZA et al., 2017), algunos estudios señalan que su consumo reduciría el riesgo de enfermedades inflamatorias, metabólicas y mejora de la sensibilidad a la insulina (KRATZ et al., 2014). Además, 
actúa como un importante señalizador de reacciones metabólicas en adipocitos (PASSOS et al., 2016).

En estudios realizados con larvas colectados del medio natural, han reportado ácidos grasos pertenecientes a la familia omega 6 y omega 3, como son los ácidos grasos esenciales linoleico y alpha linolénico, en concentraciones semejantes $(1,93$ y $1,05 \%)$ (SANCHO et al., 2015), e inferiores $(1,10$ y $0,30 \%$ ) (VARGAS et al., 2013). Así también, Gbogouri et al. (2013), reporta concentraciones superiores para ambos ácidos grasos con 4,30 y $1,40 \%$, respectivamente.

En este estudio, el aceite de larva sometida a la dieta de $M$. flexuosa (Tabla 4), presento un mayor contenido de estos. La presencia de estos dos ácidos grasos eleva la importancia nutritiva de la larva de $R$. palmarum, pues estos dos ácidos grasos, el ser humano no los puede sintetizar y tienen que ser adquiridos mediante la dieta, y son necesarios para el normal desarrollo del ser humano, especialmente en los primeros años de vida, cumpliendo diversas funciones de tejido
(MICHAELSEN et al., 2009). Además, a partir de estos dos ácidos grasos esenciales pueden ser sintetizados los demás ácidos grasos esenciales de la familia omega 6 y omega 3 (FABIAN et al., 2015).

Para la prevención de enfermedades cardiovasculares, se recomienda su consumo en una proporción $n-6 / n-3$ de $4: 1$ o menos (FOGANG MBA et al., 2017). La relación n-6/n-3 en el aceite de larva sometida a la dieta de $J$. digitata es la más próxima a lo recomendado. Esto demuestra el potencial que la larva de $R$. palmarum, no solo desde el punto de vista nutritivo, sino también ayudaría a la prevención de enfermedades cardiovasculares.

En la Amazonía peruana, pobladores rurales y grupos indígenas utilizan el aceite de esta larva para tratar enfermedades respiratorias (tos, asma, resfriados, tuberculosis, entre otros). Según Delgado et al. (2019), estos atributos estarían relacionados con la presencia de estos ácidos grasos esenciales (linoleico y alpha linolénico).

Tabla 4. Composición de ácidos grasos del aceite de larva de $R$. palmarum.

\begin{tabular}{|c|c|c|c|}
\hline \multirow{2}{*}{ Ácidos grasos (\%) } & \multicolumn{3}{|c|}{ Dieta } \\
\hline & M. flexuosa & O. bataua & J. digitata \\
\hline 08:0 Caprílico & - & - & 0,26 \\
\hline 10:0 Cáprico & - & - & 0,29 \\
\hline 12:0 Láurico & - & - & 0,28 \\
\hline 14:0 Mirístico & $2,08 \pm 0,028 b$ & $2,11 \pm 0,000 b$ & $2,56 \pm 0,014 a$ \\
\hline 16:0 Palmítico & $41,81 \pm 0,877 b$ & $44,56 \pm 0,240 \mathrm{a}$ & $43,91 \pm 0,375 a b$ \\
\hline 16:1 Palmitoleico & $1,45 \pm 0,007 b$ & $1,34 \pm 0,021 \mathrm{c}$ & $1,72 \pm 0,014 a$ \\
\hline 17:0 Heptadecaenoico & 0,26 & - & - \\
\hline 18:0 Esteárico & $5,95 \pm 0,255 b$ & $8,13 \pm 0,106 a$ & $5,16 \pm 0,064 c$ \\
\hline 18:1w-9 Oleico & $43,54 \pm 0,248 a$ & $42,06 \pm 0,276 b$ & $43,01 \pm 0,057 \mathrm{a}$ \\
\hline 18:2w-6 Linoleico & $1,96 \pm 0,028 \mathrm{a}$ & $0,67 \pm 0,007 \mathrm{c}$ & $0,76 \pm 0,000 b$ \\
\hline 18:3w-3 $\alpha$-Linolénico & $1,14 \pm 0,007 a$ & $0,43 \pm 0,035 b$ & $0,29 \pm 0,007 \mathrm{c}$ \\
\hline 20:0 Araquídico & $0,72 \pm 0,007 \mathrm{a}$ & $0,72 \pm 0,000 \mathrm{a}$ & $0,58 \pm 0,007 b$ \\
\hline Total MUFA & $44,99 \pm 0,180 \mathrm{a}$ & $43,40 \pm 0,210 b$ & $44,73 \pm 0,050 b$ \\
\hline Total PUFA & $3,10 \pm 0,025 a$ & $1,10 \pm 0,030 b$ & $1,05 \pm 0,005 b$ \\
\hline$n-6 / n-3$ & $1,72 \pm 0,010 b$ & $1,56 \pm 0,080 b$ & $2,62 \pm 0,045 a$ \\
\hline Total saturados (AGS) & $50,82 \pm 0,335 c$ & $55,51 \pm 0,255 a$ & $53,03 \pm 0,200 b$ \\
\hline Total insaturados (AGI) & $48,08 \pm 0,205 a$ & $44,48 \pm 0,240 c$ & $45,77 \pm 0,045 b$ \\
\hline AGS/AGI & $1,06 \pm 0,012 \mathrm{c}$ & $1,25 \pm 0,001 \mathrm{a}$ & $1,16 \pm 0,006 b$ \\
\hline
\end{tabular}

Datos expresados en promedio \pm desviación estándar.

Medias seguidas de letras iguales entre filas no son significativamente diferentes por la prueba de Tukey al $5 \%$ de probabilidad.

MUFA: ácidos grasos monoinsaturados; PUFA: ácidos grasos poliinsaturados.

En cuanto al contenido de ácidos grasos saturados e insaturados, el perfil de ácidos grasos del aceite para las tres dietas mostraron un mayor porcentaje de ácidos grasos saturados, que lo reportado para larvas colectadas de estípites del medio natural (DUE et al., 2009; VARGAS et al., 2013). En este estudio el aceite de larva sometidas a la dieta de $M$. flexuosa presento menor porcentaje de ácidos grasos saturados.

Comparado los valores de grasas saturadas, monoinsaturados y poliinsaturados, el aceite de larva de $R$. Palmarum se asemeja a la grasa de la carne de res que contiene 54,44 y $2 \%$, respectivamente (ROSKOSKI, 1998). Según DeFoliart (1992), algunos insectos pueden presentar mayor contenido de ácidos grasos esenciales (linoléico y alpha linolénico) que la carne bovina. Los ácidos grasos poliinsaturados tienen funciones fisiológicas importantes y son reguladoras claves de las propiedades de la membrana celular, así también, cumplen importantes funciones estructurales y funcionales en el cerebro humano (DYALL, 2015; MÜLLER et al., 2015).

Comparando el aceite de larva de $R$. palmarum con fuentes lipídicas comunes, este mostró un porcentaje de ácidos grasos saturados e insaturados semejante al aceite de palma (BOATENG et al., 2016), y mayor porcentaje de insaturados para algunas fuentes como: aceite de coco, mantequilla, carne de res, manteca de cacao, entre otros (DORNI et al., 2018; ROSKOSKI, 1998; WALLACE, 2019; WANG; MALEKY, 2018). Este hecho hace destacar su importancia nutritiva, pudiendo ser considerada un alimento saludable, pues el consumo de alimentos ricos en ácidos grasos insaturados tiene un efecto positivo para la salud humana (CABEZAS et al., 
2016), estas características podrían ser aprovechados tanto en la industria de alimentos, cosmética y farmacéutica (DUE et al., 2009; VARGAS et al., 2013).

En la comparación de medias $(\mathrm{p}<0,05)$ de los ácidos grasos insaturados para las tres dietas. El ácido graso oleico muestra diferencia significativa para las dietas M. flexuosa-O. bataua y $O$. bataua-J. digitata. Para los ácidos grasos palmitoleico, linoleico y alpha linolénico se evidenció diferencia significativa entre las tres dietas (Tabla 4).

\section{CONCLUSIONES}

Las dietas suministradas a las larvas de Rynchophorus palmarum influyen en el tipo de ácido graso metabolizado y su concentración. La larva es fuente de los ácidos grasos monoinsaturados, oleico (omega 9) y palmitoleico (omega 7), así como de los ácidos grasos esenciales, linoleico y alpha linolénico precursores de los ácidos grasos de la familia omega 6 y omega 3 . Se podría considerar que, el aceite obtenido de las larvas sometidas a la dieta de Mauritia flexuosa es más saludable, teniendo en consideración que presenta mayor porcentaje de ácidos grasos insaturados.

\section{REFERENCIAS}

ANGEL-DAPA， M. A.; RODRÍGUEZ-JARAMILLO, C.; CÁCERES-MARTÍNEZ, C. J.; SAUCEDO, P. E. Changes in Lipid Content of Oocytes of the Penshell Atrina maura as a Criterion of Gamete Development and Quality: A Study of Histochemistry and Digital Image Analysis . Journal of Shellfish Research, v. 29, n. 2, p. 407-413, 2010.

ANTONNY, B.; VANNI, S.; SHINDOU, H.; FERREIRA, T. From zero to six double bonds: Phospholipid unsaturation and organelle function. Trends in Cell Biology, v. 25, n. 7, p. 427436, 2015. 10.1016/j.tcb.2015.03.004. .

BIGAY, J.; ANTONNY, B. Curvature, Lipid Packing, and Electrostatics of Membrane Organelles: Defining Cellular Territories in Determining Specificity. Developmental Cell, v. 23, n. 5, p. 886-895, 2012.

BOATENG, L.; ANSONG, R.; OWUSU, W. B.; STEINERASIEDU, M. Coconut oil and palm oil's role in nutrition, health and national development: A review. Ghana medical journal, v. 50, n. 3, p. 189-196, 2016.

CABEZAS-ZÁBALA, C. C.; HERNÁNDEZ-TORRES, B. C.; VARGAS-ZARATE, M. Aceites y grasas: efectos en la salud y regulación mundial. Revista de la Facultad de Medicina, v. 64, n. 4 , p. $761-8,2016$.

CALDER, P. C. Fatty acids and inflammation: The cutting edge between food and pharma. European Journal of Pharmacology, v. 668, p. S50-S58, 2011. 10.1016/j.ejphar.2011.05.085.

CARTAY, R. Entre el asombro y el asco: el consumo de insectos en la cuenca amazónica. El caso del Rhynchophorus palmarum (Coleoptera Curculionidae). revista colombiana de antropología, v. 54, n. 2, p. 143-169, 2018.

COSTA-NETO, E. M. Antropoentomofagia Insetos na Alimentação Humana. Editora UEFS, 2011. Feira de Santana.

COSTA-NETO, E. M. Insects as hum food: An overv. Amazon. Rev. Anthropol., v. 5, n. 53, p. 562-582, 2013.

DAVID SANCHO-AGUILERA, DAVID LANDÍVARVALVERDE, DIEGO SARABIA-GUEVARA, M. DE J. Á.-G. Caracterización del extracto graso de larvas de Rhynchophorus palmarum L. Ciencia y Tecnología de Alimentos Mayo, v. 25, n. 2, p. 39-44, 2015.

DEFOLIART, G. R. Insects as Human Food Gene Defoliart discusses nutritional and economic aspects. Crop Protection, v. 11, p. 395-99, 1992. 10.1007/978-981-10-1524-3_7.

DEFOLIART, G. R. Edible insects as minilivestock. Biodiversity and Conservation, v. 321, p. 306-321, 1995.

DELGADO, C.; COUTURIER, G.; MATHEWS, P.; MEJIA, K. Producción y comercialización de la larva de "Rhynchophorus palmarum" (Coleoptera: Dryophtoridae) en la Amazonía peruana. Boletín de la SEA, v. 41, n. 42, p. 407-412, 2008.

DELGADO, C.; ROMERO, R.; ESPINOZA, R. V.; TRIGOZO, M.; CORREA, R. Rhynchophorus palmarum used in Traditonal Medicine in the Peruvian Amazon. Ethnobiology Letters, v. 10, n. 1, p. 120-128, 2019.

DORNI, C.; SHARMA, P.; SAIKIA, G.; LONGVAH, T. Fatty acid profile of edible oils and fats consumed in India. Food Chemistry, v. 238, p. 2018. 10.1016/j.foodchem.2017.05.072.

DUE, E. A.; ZABRI, H. C. B. L.; KOUADIO, J. P. E. N.; KOUAME, L. P. Fatty acid composition and properties of skin and digestive fat content oils from Rhynchophorus palmarum $\mathrm{L}$. larva. African Journal of Biochemistry Research, v. 3, n. 4, p. 089-094, 2009.

DYALL, S. C. Long-chain omega-3 fatty acids and the brain: A review of the independent and shared effects of EPA, DPA and DHA. Frontiers in Aging Neuroscience, v. 7, n. 52, p. 1-15, 2015.

ESHAK, E. S.; ISO, H.; YAMAGISHI, K.; CUI, R.; TAMAKOSHI, A. Dietary intakes of fat soluble vitamins as predictors of mortality from heart failure in a large prospective cohort study. Nutrition, v. 47, p. 50-55, 2018. 10.1016/j.nut.2017.09.009

FABIAN, C. J.; KIMLER, B. F.; HURSTING, S. D. Omega-3 fatty acids for breast cancer prevention and survivorship. Breast Cancer Research, v. 17, n. 1, p. 1-11, 2015.

FAPESP. Insetos comestíveis. 2020. Disponível em: 
<file:///C:/Users/jc/AppData/Local/Temp/060-

067_entomofagia_290-2.pdf >. .

FINKE, M. D.; OONINCX, D. Insects as Food for Insectivores. Elsevier, 2013.

FOGANG MBA, A. R.; KANSCI, G.; VIAU, M.; et al. Lipid and amino acid profiles support the potential of Rhynchophorus phoenicis larvae for human nutrition. Journal of Food Composition and Analysis, v. 60, p. 64-73, 2017. 10.1016/j.jfca.2017.03.016

FOOD AND ARGRICULTURE ORGANIZATION OF THE UNITED NATIONS. Edible insects. Future prospects for food and feed security. 2013.

GBOGOURI, G. A.; BEUGRE, G. A. M.; BROU, K.; ATCHIBRI, O. A.; LINDER, M. Rhynchophorus palmarum L. larva, an edible insect in Côte d'Ivoire: Nutritional value and characterization of the lipid fraction. International Journal of Chemical Sciences, v. 11, n. 4, p. 1692-1704, 2013.

GIBLIN-DAVIS, R. M.; GERBER, K.; GRIFFITH, R. Laboratory Rearing of Rhynchophorus cruentatus and $R$. palmarum (Coleoptera: Curculionidae). The Florida Entomologist, 1989.

JORIS, P. J.; MENSINK, R. P. Role of cis-Monounsaturated Fatty Acids in the Prevention of Coronary Heart Disease. Current Atherosclerosis Reports, v. 18, n. 7, p. 38, 2016. Current Atherosclerosis Reports. 10.1007/s11883-016-0597-y

KAHM, F.; MEJÍA, K. Las palmeras nativas de importancia economica en La amazonia peruana. Folia Amazónica, v. 1, n. 1-2, p. 103-116, 2006.

KRATZ, M.; MARCOVINA, S.; NELSON, J. E.; et al. Dairy fat intake is associated with glucose tolerance, hepatic and systemic insulin sensitivity, and liver fat but not $\beta$-cell function in humans. American Journal of Clinical Nutrition, v. 99, n. 6 , p. 1385-1396, 2014.

MEXZÓN, R. G.; CHINCHILLA, C. M.; CASTRILlO, G.; DANNY, S. Biología y hábitos de Rhynchophorus palmarum L. asociado a la palma aceitera en Costa Rica. ASD Oil Palm Papers, v. 8, n. 8, p. 14-21, 1994.

MICHAELSEN, K. F.; HOPPE, C.; ROOS, N.; et al. Choice of foods and ingredients for moderately malnourished children 6 months to 5 years of age. Food and Nutrition Bulletin, v. 30, n. 3, p. S343-S404, 2009.

MÜLLER, C. P.; REICHEL, M.; MÜHLE, C.; et al. Brain membrane lipids in major depression and anxiety disorders. Biochimica et Biophysica Acta - Molecular and Cell Biology of Lipids, v. 1851, n. 8, p. 1052-1065, 2015. 10.1016/j.bbalip.2014.12.014

PASSOS, M. E. P.; ALVES, H. H. O.; MOMESSO, C. M.; et al. Differential effects of palmitoleic acid on human lymphocyte proliferation and function. Lipids in Health and Disease, v. 15, n. 1, p. $1-11,2016.10 .1186 / \mathrm{s} 12944-016-0385-2$

QUEIROZ, A.; DE ALMEIDA, S.; KRUG, C.; ALVES, W. A. Manejo de Rhynchophorus palmarum em Campo de Produção de Sementes de Palma de Óleo. Manaus, AM, 2013.

ROSKOSKI, R. Bioquimica. McGraw-Hill, 1998. México.

RUIZ, C.; DÍAZ, C.; ANAYA, J.; ROJAS, R. Análisis proximal, antinutrientes, perfil de ácidos grasos y de aminoácidos de semillas y tortas de 2 especies de sacha inchi (Plukenetia volubilis y Plukenetia huayllabambana). Revista de la Sociedad Química del Perú, v. 79, n. 1, p. 29-36, 2013.

RUMPOLD, B. A.; SCHLÜTER, O. K. Nutritional composition and safety aspects of edible insects. Molecular Nutrition and Food Research, v. 57, n. 5, p. 802-823, 2013.

SANTANA, C. C.; NASCIMENTO, J. S.; DE COSTA, M. M.; et al. Avaliação do desenvolvimento e reservas energéticas de larvas de Rhynchophorus palmarum (Coleoptera:Curculionidae) em diferentes dietas. Revista Brasileirade Ciencias Agrarias, v. 9, n. 2, p. 205-209, 2014.

SIMOPOULOS, A. P. An increase in the Omega-6/Omega-3 fatty acid ratio increases the risk for obesity. Nutrients, v. 8, n. 3, p. 1-17, 2016.

SOUZA, C. O.; TEIXEIRA, A. A. S.; BIONDO, L. A.; et al. Palmitoleic acid reduces the inflammation in LPS-stimulated macrophages by inhibition of $\mathrm{NF} \kappa \mathrm{B}$, independently of PPARs. Clinical and Experimental Pharmacology and Physiology, v. 44, n. 5, p. 566-575, 2017. 10.1111/1440-1681.12736

TZOMPA-SOSA, D. A.; YI, L.; VAN VALENBERG, H. J. F.; VAN BOEKEL, M. A. J. S.; LAKEMOND, C. M. M. Insect lipid profile: Aqueous versus organic solvent-based extraction methods. Food Research International, v. 62, p. 1087-1094, 2014. 10.1016/j.foodres.2014.05.052

VAFEIADOU, K.; WEECH, M.; ALTOWAIJRI, H.; et al. Replacement of saturated with unsaturated fats had no impact on vascular function but beneficial effects on lipid biomarkers, E-selectin, and blood pressure: Results from the randomized, controlled Dietary Intervention and VAScular function (DIVAS) study. American Journal of Clinical Nutrition, v. 102, n. 1, p. 40-48, 2015.

VALENZUELA, A.; SUSANA, N. Ácidos grasos omega-6 y omega-3 en la nutrición perinatal: su importancia en el desarrollo del sistema nervioso y visual. Rev Chil Pediatr, v. 74, p. 149-57, 2003.

VARGAS, G. E.; ESPINOZA, G.; RUIZ, C.; ROJAS, R. Valor nutricional de la Larva de Rhynchophorus palmarum L : Comida tradicional en la amazonía peruana. Revista de la sociedad química del Perú, v. 79, n. 1, p. 64-70, 2013. 
VELDKAMP, T.; VAN DUINKERKEN, G.; VAN HUIS, A.; et al. Insects as a sustainable feed ingredient in pig and poultry diets-a feasibility study. Food Chemistry, v. 50, p. 192-195, 2012.

VAN HUIS, A. Potential of Insects as Food and Feed in Assuring Food Security. Annual Review of Entomology, v. 58, n. 1, p. 563-583, 2013.

VAN HUIS, A. Environmental Sustainability of Insects as Human Food. Reference Module in Food Science, p. 1-5, 2019. 10.1016/B978-0-08-100596-5.22589-4

WALLACE, T. C. Health Effects of Coconut Oil-A Narrative Review of Current Evidence. Journal of the American College of Nutrition, v. 38, n. 2, p. 97-107, 2019. Taylor \& Francis. $10.1080 / 07315724.2018 .1497562$

WANG, H.; MALEKY, F. Effects of cocoa butter triacylglycerides and minor compounds on oil migration. Food Research International, v. 106, p. 213-224, 2018. 10.1016/j.foodres.2017.12.057

WATANABE, Y.; TATSUNO, I. Prevention of Cardiovascular Events with Omega-3 Polyunsaturated. The official journal of the Japan Atherosclerosis Society and the Asian Pacific Society of Atherosclerosis and Vascular Diseases, v. 26, p. 183-198, 2019.

WEYLANDT, K. H.; CHIU, C. Y.; GOMOLKA, B.; WAECHTER, S. F.; WIEDENMANN, B. Omega-3 fatty acids and their lipid mediators: Towards an understanding of resolvin and protectin formation. Omega-3 fatty acids and their resolvin/protectin mediators. Prostaglandins and Other Lipid Mediators, v. 97, n. 3-4, p. 73-82, 2012. 10.1016/j.prostaglandins.2012.01.005

YEHUDA, S.; RABINOVITZ, S.; CARASSO, R. L.; MOSTOFSKY, D. I. The role of polyunsaturated fatty acids in restoring the aging neuronal membrane. Neurobiology of Aging, v. 23, n. 5, p. 843-853, 2002. 